\title{
A study to evaluate gestational age with the help of placental thickness
}

\section{Aditi Tiwari*, Kavita Chandnani}

Department of Obstetrics and Gynecology, SBKS Medical Institute and Research Centre, Piparia, Vadodara, Gujarat, India

Received: 14 August 2013

Accepted: 5 September 2013

\author{
*Correspondence: \\ Dr. Aditi Tiwari, \\ E-mail: tiwariaditi085@gmail.com
}

(C) 2013 Tiwari A et al. This is an open-access article distributed under the terms of the Creative Commons Attribution Non-Commercial License, which permits unrestricted non-commercial use, distribution, and reproduction in any medium, provided the original work is properly cited.

\begin{abstract}
Background: To assess relationship of placental thickness with gestational age.

Methods: The study was conducted on 754 normal antenatal patients, of all gestational ages attending the out patient department of Dhiraj hospital from October 2012 to March 2013. USG was done by using Philips HD 7 machine with a $3.75 \mathrm{MHz}$ sector probe. After estimating the fetal age by CRL, BPD, HC, AC, and FL, the placental thickness with standard deviation was calculated for all gestational ages.

Results: It was observed that the placental thickness gradually increased from $15 \mathrm{~mm}$ at 11 weeks of gestation to 36.3 $\mathrm{mm}$ at 39 weeks. From the $22 \mathrm{nd}$ week to the 35 th week of gestation the placental thickness coincide almost exactly with the gestational age in weeks.

Conclusion: Placental thickness is a good parameter for estimation of gestational age especially in the late second trimester and early third trimester.
\end{abstract}

Keywords: Placental thickness, Gestational age

\section{INTRODUCTION}

The best possible ante partum care and the successful deliveries of babies always revolve around the accurate knowledge of the gestational age. ${ }^{4}$ The gestational age is approximately 280 days, which is calculated from the first day of the last menstrual period and so, the dating of the pregnancy starts even before the fertilization. The determination of the gestational age is a common clinical problem. Ultrasonography is commonly used to estimate the gestational age by measuring the foetal dimensions like the Biparietal Diameter (BPD), the Abdominal Circumference (AC), the Head Circumference (HC) and the Femur Length (FL). An ultrasonograph is prone to observer bias, as it depends on the observers' technical skills. Also, the foetal parameters, the different techniques of measurement and the positional problems may diminish the accuracy of the gestational age estimation. ${ }^{6}$ Wolfson et al., showed that the biparietal diameter was not reliable in the foetuses which had a premature rupture of the membranes. ${ }^{11}$

There are some drawbacks in those above said parameters in estimating the gestational age. So, there is a need of another parameter for supplementing the gestational age estimation with minimal error. Nyberg and Finberg reported that the placental thickness parallels the gestational age. ${ }^{10}$

At term, placenta is discoid with a diameter of 15 to 25 cms and approximately $3 \mathrm{cms}$ thick and weighs about 500 to 600 grams. ${ }^{10}$ Placental thickness increases with the age of the foetus. ${ }^{1,2,3,8}$

Therefore the thickness in $\mathrm{mm}$ can approximate the gestational age (in weeks) $+/-10 \mathrm{~mm}$. The maximum thickness considered normal at any stage in pregnancy is often taken at $4 \mathrm{cms}^{2}$ The present study was undertaken 
to evaluate the relationship between placental thickness and gestational age of the fetus.

\section{METHODS}

Present study was conducted in the department of Gynaecology and Obstetrics, Dhiraj Hospital, S.B.K.S.MIRC, Piparia, Vadodara, Gujarat.

754 antenatal cases of all gestational ages (> 10 weeks of gestation) were selected in this study. Patients with gestational hypertension, gestational diabetes mellitus, intra uterine growth restriction, hydrops fetalis, congenital malformation, multiple pregnancy were excluded from the study. The ultrasonography was done in the outpatient department with Philips HD 7 machine with a $3.75 \mathrm{MHz}$ sector probe.

The patients were scanned with a full bladder in a supine position. The fetus was examined for viability, foetal congenital abnormalities and various growth parameters. The gestational age was determined by measuring the CRL up to the 11th week, by BPD and FL in the second trimester and BPD, FL, AC in the third trimester.

The placenta was then localized in the longitudinal section and the placental thickness was measured near the insertion of the umbilical cord.

\section{RESULTS}

The mean values of placental thickness along with respective standard deviation (SD) were calculated for different gestational ages from the 11th week to the 39th week.

The results of the study have been shown in table 1 .

Table 1: Placental thickness.

\begin{tabular}{|lll|}
\hline $\begin{array}{l}\text { Gestational age } \\
\text { (in weeks) }\end{array}$ & $\begin{array}{l}\text { Number } \\
\text { of cases }\end{array}$ & $\begin{array}{l}\text { Placental } \\
\text { thickness(mm) } \\
\text { Mean +/- SD }\end{array}$ \\
\hline 10 & 19 & $15+/-2.9$ \\
\hline 11 & 18 & $15.2+/-3.0$ \\
\hline 12 & 16 & $15.4+/-3.1$ \\
\hline 13 & 11 & $16.6+/-2.9$ \\
\hline 14 & 18 & $16.9+/-3.6$ \\
\hline 15 & 18 & $17.7+/-3.2$ \\
\hline 16 & 25 & $20.1+/-2.3$ \\
\hline 17 & 22 & $20.5+/-2.9$ \\
\hline 18 & 21 & $22.2+/-4.0$ \\
\hline 19 & 12 & $22.4+/-2.8$ \\
\hline
\end{tabular}

\begin{tabular}{|c|c|c|}
\hline 20 & 16 & $22.6+/-2.7$ \\
\hline 21 & 10 & $23.5+/-3.8$ \\
\hline 22 & 17 & $23.8+/-3.2$ \\
\hline 23 & 17 & $24.2+/-3.2$ \\
\hline 24 & 10 & $25.0+/-3.5$ \\
\hline 25 & 11 & $26.8+/-3.5$ \\
\hline 26 & 18 & $27.6+/-2.9$ \\
\hline 27 & 22 & $27.8+/-1.8$ \\
\hline 28 & 25 & $28.5+/-4.6$ \\
\hline 29 & 26 & $29.8+/-4.0$ \\
\hline 30 & 50 & $30.1+/-2.2$ \\
\hline 31 & 50 & $32.0+/-3.1$ \\
\hline 32 & 63 & $32.5+/-3.0$ \\
\hline 33 & 42 & $32.6+/-2.5$ \\
\hline 34 & 56 & $33.0+/-3.1$ \\
\hline 35 & 31 & $33.0+/-2.9$ \\
\hline 36 & 29 & $33.2+/-2.6$ \\
\hline 37 & 38 & $34.8+/-3.2$ \\
\hline 38 & 30 & $36.1+/-2.5$ \\
\hline 39 & 13 & $36.3+/-2.3$ \\
\hline
\end{tabular}

In our study, up to 21 weeks of gestation the mean placental thickness was slightly higher than the gestational age (1-4 mm). From the $22 \mathrm{nd}$ week to the 35 th week of gestation the placental thickness almost matched the gestational age in weeks, thereafter the placental thickness was lower by (1-2 mm) [Table 1].

Total cases: 754 .

\section{DISCUSSION}

The result of our study showed that placental thickness increased linearly with the advancing gestational age. This means that placental thickness can be used as an indicator of gestational age.

Kunlmann $^{5}$ and Warsoff et al in 1996 reported that placental thickness of less than $2.5 \mathrm{cms}$ is associated with intra-uterine growth restriction, while thick placentas are associated with diabetes mellitus, hydrops fetalis, intrauterine infections.

Early reports of placental localization by ultrasound examination were published by Donald (1968), Kobayashi (1970) and Gottesfield (1966). Nyberg and 
Finberg (1990) also reported that placental thickness in millimeter parallel gestational age in weeks.

\section{REFERENCES}

1. Anupama J, Ganesh K, Agrawal U, Khakharwal S (2001). Placental thickness: a sonographic indicator of gestational age. J obst. Gyne. Indian 51(3): 48-49.

2. Hoddick WK, Mahony BS, Callen PW, Filly RA (1985). Placental thickness. J. Ultra Med. 4(9): 479482.

3. Jauniaux E, Ramsay B, Campbell S (1994). Ultrasonographic investigation of placental morphologic characteristics and size during the second trimester of pregnancy. Am. J. obst. Gyn. 160(1 pt1): 130-137.

4. Journal of clinical diagnosis and research, 2012 December; 6(10): 1732-1735.

5. Kunlmann RS, Warsof S. Ultrasound of the placenta Clinical African Journal Obstetric \& Gynecology 1996; 39:34.

6. Malhotra N, Kumar P. Ultrasound in Obstetrics and Gynaecology. 3rd ed. Mumbai: Jaypee Brothers Medical Publishers Private Ltd; 1999. Chapter 11,
Measurement of foetal parameters; Chapter 27, Ultrasound and admission test in labour. Chapter 55, Pitfalls in USG;pp92-98,198-200,386-88.

7. Mathai Betty M, Singala Subhash C, Nittala Pramod P, Chakravarti Rajesh J, Toppo Julius N, J. of obstetrics and gyanaecology of India 2013 march12.

8. Mital P, Pooja N, Mehndriatta K (2002). Placental thickness: a sonograpgic parameter of estimating gestational age of the foetus. Indian journal of radiol. Imaging 12: 553-554.

9. Nyberg DA, Finberg HJ. The placenta, placental membranes and umbilical cord. Journal on diagnostic ultrasound of foetal anomalies. 1990;21(4):623-75.

10. Sadler TW (2004). Langman's medical embryology $11^{\text {th }}$ edition. Baltimore, MD: Lippincott Williams and Wilkins p 112- 91.

11. Wolfson RN, Zador IE, Havorsen P, Andrews B, Sokol RJ. Biparietal diameter in premature rupture of membranes: Errors in estimating gestational age. J Clin Ultrasound. 1983;11:371-74.

DOI: $10.5455 / 2320-1770$. ijrcog20131202

Cite this article as: Tiwari A, Chandnani K. A study to evaluate gestational age with the help of placental thickness. Int J Reprod Contracept Obstet Gynecol 2013;2:503-5. 